\title{
On the algebraic non-integrability of the Halphen system
}

\author{
Andrzej J. MACIEJEWSKI \\ Institute of Astronomy, N. Copernicus University \\ Chopina 12-18, 87-100 Toruń, Poland \\ (e-mail: maciejka@astri.uni.torun.pl ) \\ and \\ JEAN-MARIE STRELCYN \\ Département de Mathématiques, Université de Rouen, \\ 76821 Mont Saint Aignan Cedex, France, URA CNRS 1378 \\ (e-mail: strelcyn@univ-rouen.fr) \\ and \\ Laboratoire Analyse, Géométrie et Applications, URA CNRS 742, \\ Institut Galilée, Département de Mathématiques, \\ Avenue J.-B.Clément, 93430 Villetaneuse, France \\ (e-mail: strelcyn@math.univ-paris13.fr)
}

November 3, 2018

\begin{abstract}
It is proved that the Halphen system of ordinary differential equations has no non-trivial rational first integrals.
\end{abstract}

\section{Introduction}

different classes of systems of ordinary differential equations (in abbreviation ODE) is again of great actuality. In [14] an algebraic method of proving the non-integrability of polynomial systems of ODE with homogeneous right sides of the same degree was presented and tested on some non-trivial examples (see also [15] and [16] for another applications). This method, according

to the best of our knowledge, was presented for the first time in the fundamental book of J.-P. Jouanolou [10]. Indeed, on pages 193-195 of [10], M.H.A. Levelt, 
the referee of the book, proved using this method that the Jouanolou system of ODEs

$$
\frac{d x}{d t}=z^{s}, \quad \frac{d y}{d t}=x^{s}, \quad \frac{d z}{d t}=y^{s} ; \quad s \in \mathbb{N}, \quad \sim \geq \not \models
$$

definition). In fact, the basic ideas of the method were already introduced by M.N. Lagutinskii in his pioneering, but, unfortunately, completely unknown works [12, 13]. See [4, 5], where one can find more details on M.N. Lagutinskii and his works on integrability which are direct continuation of the seminal Darboux paper [3].

In this note, we study the Halphen system of ODEs [8, 9] defined on $\mathbb{C}^{\nVdash}$ (or $\left.\mathbb{R}^{\nVdash}\right)$ :

$$
\left.\begin{array}{l}
\frac{d x_{1}}{d t}=x_{2} x_{3}-x_{1}\left(x_{2}+x_{3}\right), \\
\frac{d x_{2}}{d t}=x_{3} x_{1}-x_{2}\left(x_{3}+x_{1}\right), \\
\frac{d x_{3}}{d t}=x_{1} x_{2}-x_{3}\left(x_{1}+x_{2}\right),
\end{array}\right\}
$$

Theorem The Halphen system (1) does not admit any non-trivial rational first integral.

To do this we first apply the Lagutinskii-Levelt procedure that allows to state non-existence of a polynomial first integral. To finish the proof it was necessary to supplement the method by more subtle investigations where specific properties of the Halphen system are crucial.

It is well known that for the polynomial systems of ODE the non existence of a non-trivial rational first integral is equivalent to the non-existence of an algebraic first integral. This is a consequence of Bruns theorem [6, vol.III, chap. XVII], [11]. For

polynomial systems with the homogeneous right sides of the same degree, called the homogeneous systems, the non-existence of a

rational first integral is equivalent to the non-existence of a meromorphic first integral [21]. Consequently,

the Halphen system (11) does not admit any non-trivial

algebraic or meromorphic first integral.

The Halphen system appears in different contexts, e.g., as a result of the self-dual Yang-Mills reduction [2], [18], [19], or, in general relativity,

in the study of $S U(2)$-invariant four metrics (Bianchi IX metrics) [17], [1].

It is worth noticing that, in spite of the lack of meromorphic first integrals, the Halphen system can be explicitly integrated - we can express its general solution 
in terms of elliptic integrals with 'variable modulus' (see [8], [18], [17]). Moreover, as it was mentioned in [19] and [20], the first integrals of the Halphen system do

indeed exist, although they are not global and are multi-valued non-algebraic functions. See also [7] for discussion of special properties of the Halphen system.

The paper is organized as follows. In Sec. 2 we gather all necessary algebraic facts and present the basic steps of the Lagutinskii-Levelt procedure. The proof of our theorem is given in Sec. 3 .

\section{$2 \quad$ Algebraic preliminaries}

$$
\frac{d x_{i}}{d t}=V_{i}\left(x_{1}, \ldots, x_{n}\right), \quad 1 \leq i \leq n,
$$

Here, as usual, we denote by $\mathbb{K}\left[\curvearrowleft_{\nVdash}, \ldots, \curvearrowleft_{\ltimes}\right]$ the polynomial ring in $n$ variables $x_{i}, 1 \leq i \leq n$, with coefficients in a commutative field $\mathbb{K}$, and by $\mathbb{K}\left(\curvearrowleft_{\nVdash}, \ldots, \curvearrowleft_{\ltimes}\right)$ the field of rational fractions of $n$ variables with coefficients from $\mathbb{K}$. Throughout this note we assume that $\mathbb{K}=\mathbb{C}$.

A function $F$ is a first integral of system (2) if it satisfies the following equation

$$
d_{V}(F) \stackrel{\text { def }}{=} \sum_{i=1}^{n} V_{i} \partial_{i} F=0, \quad \text { where } \quad \partial_{i} F=\frac{\partial F}{\partial x_{i}} .
$$

notion of derivations is used. A derivation $d$ is a linear mapping of $\mathbb{K}\left[\curvearrowleft_{\nVdash}, \ldots, \curvearrowleft_{\star}\right]$ (or of $\mathbb{K}\left(\curvearrowleft_{\nVdash}, \ldots, \curvearrowleft_{\ltimes}\right)$ ) into itself satisfying the Leibnitz rule $d_{V}(F G)=G d_{V}(F)+$ $F d_{V}(G)$. Thus, we can talk about derivation $d_{V}$ instead of system (2). The derivation $d_{V}$ is called homogeneous if the corresponding system of ODE (2) is homogeneous.

The main object in our investigation are the so called Darboux polynomials of a derivation $d_{V}$ (or partial first integrals of a system of ODEs (2)), i.e., polynomials $F \in \mathbb{C}\left[\curvearrowleft_{\nVdash}, \ldots, \curvearrowleft_{\ltimes}\right] \backslash \mathbb{C}$ such that

$$
d_{V}(F)=\sum_{i=1}^{n} V_{i} \partial_{i} F=P F,
$$

Darboux polynomial $F$ is nothing else but a first integral of the system of ODEs called also a constant of the derivation $d_{V}$. These polynomials, as an investigation tool of the integrability of the system (2), were introduced for the first time by Darboux in [3].

It is easy to prove the following facts (see [14]).

1. An element $F=A / B \in \mathbb{C}\left(\curvearrowleft_{\nVdash}, \ldots, \curvearrowleft_{\ltimes}\right)$, with relatively prime polynomials $A, B \in \mathbb{C}\left[\curvearrowleft_{\nVdash}, \ldots, \curvearrowleft_{\alpha}\right]$ is a first integral of (2) if and only if $A$ and $B$ are Darboux polynomials with the same 'eigenvalue' $P$, i.e., $d_{V}(A)=P A$, and $d_{V}(B)=P B$. 
2. If $F \in \mathbb{C}\left[\curvearrowleft_{\nVdash}, \ldots, \curvearrowleft_{\ltimes}\right]$ is a Darboux polynomial of (2), then all its irreducible factors are also Darboux polynomials.

3. The finite product of Darboux polynomials is also a Darboux polynomial. More precisely,

$$
\text { if } \quad d_{V}\left(F_{i}\right)=P_{i} F_{i}, \quad 1 \leq i \leq s, \quad \text { then } \quad d_{V}\left(\prod_{i=1}^{s} F_{i}\right)=\left(\sum_{i=1}^{s} P_{i}\right)\left(\prod_{i=1}^{s} F_{i}\right) .
$$

4. For a homogeneous derivation $d_{V}$ (or a homogeneous system) if $d_{V}(F)=$ $P F$ for some $F, P \in \mathbb{C}\left[\curvearrowleft_{\nVdash}, \ldots, \curvearrowleft_{\ltimes}\right]$ then also $d_{V}\left(F^{+}\right)=P^{+} F^{+}$, where by $G^{+}$we denote the homogeneous component of the highest degree of a polynomial $G$.

5. For homogeneous derivations $d_{V}$ (or homogeneous systems), the following assertion holds [14, Lemma 2.1]. If $F$ is a Darboux polynomial, with eigenvalue $P$ such that $d_{V}(F)=P F$, then $P$ is a homogeneous polynomial, and all homogeneous components of $F$ are also Darboux polynomials corresponding to the same $P$.

6. Let us assume that $F_{i}$, for $1 \leq i \leq s$, are all (up to a multiplicative constant) irreducible homogeneous Darboux polynomials of a homogeneous derivation $d_{V}$, and let $d_{V}\left(F_{i}\right)=P_{i} F_{i}$, for $1 \leq i \leq s$. In such a case if the polynomials $P_{i}$, for $1 \leq i \leq s$, are linearly independent over $\mathbb{Z}$, then any Darboux polynomial of $d_{V}$ is (up to a multiplicative constant) of the form $\prod_{i=1}^{s} F_{i}^{\alpha_{i}}$, where $\alpha_{i}, 1 \leq i \leq s$, are non negative integers. This assertion easily follows from properties 2,3 and 5 given above.

Thus, the non-existence of non constant Darboux polynomials implies the non-existence of rational first of integrals of the corresponding system of ODEs. However, there exist systems with Darboux polynomials that have no rational first integral. We will show that the Halphen system belongs to this class of systems.

Now, let us present the basic steps of the Lagutinskii-Levelt procedure. Let us consider the system (2) with homogeneous right hand sides of the same degree $k$. To prove the non-existence of a polynomial first integral or Darboux polynomial we make use of Darboux points, i.e., the points $z=\left(z_{1}, \ldots, z_{n}\right) \neq(0, \ldots, 0)$ satisfying the following equations

$$
V_{i}(z)=\lambda z_{i}, \quad 1 \leq i \leq n, \quad \text { for some } \quad \lambda \in \mathbb{C} .
$$

Note that the existence of a Darboux point $z$ for the system is equivalent to the existence of the straight line solution of the form

$$
x_{i}(t)=z_{i} \phi(t), \quad 1 \leq i \leq n, \quad \text { where } \quad \frac{d \phi}{d t}=\lambda \phi^{k} .
$$


Now, let us assume that a homogeneous polynomial $F$ of degree $m \geq 1$ is a Darboux polynomial of our system:

$$
\sum_{i=1}^{n} V_{i} \partial_{i} F=P F
$$

Combining the above equation with the Euler identity

$$
\sum_{i=1}^{n} x_{i} \partial_{i} F=m F
$$

we can eliminate one partial derivative of $\mathrm{F}$, e.g., $\partial_{n} F$, and we obtain the equation

$$
\sum_{i=1}^{n-1}\left(V_{i} x_{n}-V_{n} x_{i}\right) \partial_{i} F=\left(x_{n} P-m V_{n}\right) F
$$

Without any loss of generality, we can assume that the last component of a chosen Darboux point $z$ does not vanish, and we set $z_{n}=1$. Putting $x_{n}=1$ in the equation (3) and shifting the origin to the Darboux point by the transformation

$$
x_{i}=y_{i}+z_{i}, \quad 1 \leq i \leq n-1,
$$

we obtain the following equation

$$
\sum_{i=1}^{n-1} w_{i} \partial_{i} f=q f
$$

where

$$
\left.\begin{array}{c}
w_{i}=w_{i}\left(y_{1}, \ldots, y_{n-1}\right)=v_{i}\left(y_{1}, \ldots, y_{n-1}\right)-v_{n}\left(y_{1}, \ldots, y_{n-1}\right)\left(y_{i}+z_{i}\right), \\
q=p\left(y_{1}, \ldots, y_{n-1}\right)-m v_{n}\left(y_{1}, \ldots, y_{n-1}\right), \quad \partial_{i}=\frac{\partial}{\partial y_{i}}, \quad 1 \leq i \leq n-1,
\end{array}\right\}
$$

and where for an arbitrary polynomial $G\left(x_{1}, \ldots, x_{n}\right)$ we define

$$
g\left(y_{1}, \ldots, y_{n-1}\right) \stackrel{\text { def }}{=} G\left(y_{1}+z_{1}, \ldots, y_{n-1}+z_{n-1}, 1\right)
$$

As $z$ is a Darboux point, then all the polynomials $w_{i}, 1 \leq i \leq n-1$, vanish at the origin $y=0$. Comparing the minimal degree terms of both sides in (四) we obtained that

$$
\sum_{i=1}^{n-1} l_{i} \frac{\partial h}{\partial y_{i}}=\chi h
$$

where

$$
l_{i}=\sum_{j=1}^{n-1} l_{i j} y_{j}, \quad 1 \leq i \leq n-1,
$$


are homogeneous linear terms of $w_{i}, \chi$ is the zero order term of $q$ and $h$ is the non-trivial homogeneous component of $f$ of the lowest degree. Let us denote by $\rho_{i}, 1 \leq i \leq n-1$ the eigenvalues of the matrix $\mathbf{L}=\left[l_{i j}\right]_{1 \leq i, j \leq n-1}$. Then, it can be shown (see [14, Lemma 2.3] ) that if $F$ is a Darboux polynomial of (2) then there exist non-negative integers $i_{k}, 1 \leq k \leq n-1$, such that

$$
\sum_{k=1}^{n-1} i_{k} \rho_{k}=\chi, \quad \sum_{k=1}^{n-1} i_{k}=\operatorname{deg} h \leq m .
$$

The eigenvalues of the matrix $\mathbf{L}$ we will call Lagutinskii-Levelt exponents. The proof of the non-integrability presented here uses essentially the relations (6).

In the proof of our theorem we will also use the following well known fact. Let $F \in \mathbb{C}[\curvearrowright] \backslash \mathbb{C}$ be a polynomial of a degree $s$ in one variable $y$. Let us denote by $y_{i}, 1 \leq i \leq s$, the roots of $F$ and by $\alpha_{i}, 1 \leq i \leq s$, their multiplicities respectively. As it is well known, in this case

$$
\frac{F^{\prime}}{F}=\sum_{i=1}^{s} \frac{\alpha_{i}}{y-y_{i}}
$$

where $F^{\prime}$ is the derivative of $F$ with respect to $y$. Moreover, such decomposition is unique. Indeed, if

$$
\frac{F^{\prime}}{F}=\sum_{i=1}^{p} \frac{\beta_{i}}{y-y_{i}^{\prime}},
$$

then $s=p, y_{i}=y_{i}^{\prime}, 1 \leq i \leq s$, and $\alpha_{i}=\beta_{i}, 1 \leq i \leq s$.

\section{Proof of the theorem}

The Halphen system is invariant with respect to permutations of variables. Let us denote by $\tau$ an automorphism of $\mathbb{C}\left[\curvearrowleft_{\nVdash}, \curvearrowleft_{\not \prime}, \curvearrowleft_{\nVdash}\right]$ induced by a permutation of $\left\{x_{1}, x_{2}, x_{3}\right\}$. If by $d_{H}$ we denote the derivation defined by the Halphen system then

$$
\tau \circ d_{H} \circ \tau^{-1}=d_{H} .
$$

From this invariance property of $d_{H}$ we have immediately that

$$
\text { if } \quad d_{H}(F)=P F \quad \text { then } \quad d_{H}(\tau(F))=\tau(P) \tau(F) .
$$

First we show the following

Lemma 1 The Halphen system (1) does not have any polynomial first integral.

Proof. Let us consider a Darboux point $z^{0}=(1,1,1)$ of the Halphen system and let us assume that $F \in \mathbb{C}\left[\curvearrowleft_{\nVdash}, \curvearrowleft_{\sharp}, \curvearrowleft_{\nVdash}\right] \backslash \mathbb{C}$ is a homogeneous first integral 
of degree $m$ and $F \neq 0$ (see point (4) of sec. 2). Direct calculations show that for the chosen Darboux point we have (see (5))

$$
w_{1}=-y_{1}\left(1+2 y_{2}+y_{1} y_{2}\right), \quad w_{2}=-y_{2}\left(1+2 y_{2}+y_{1} y_{2}\right), \quad q=m\left(1-y_{1} y_{2}\right) .
$$

Thus, the Lagutinskii-Levelt exponents are $\rho_{1}=\rho_{2}=-1$, and $\chi=m$. Then (see (6) ) there exist two non-negative integers $i_{1}$ and $i_{2}$ such that $i_{1} \rho_{1}+i_{2} \rho_{2}=$ $-\left(i_{1}+i_{2}\right)=m$ but $m>0$. Contradiction.

Although the Halphen system has no polynomial first integrals it has Darboux polynomials. If we denote

$$
\left.\begin{array}{l}
F_{1}=x_{1}-x_{2}, \\
F_{2}=x_{2}-x_{3}, \\
F_{3}=x_{3}-x_{1}, \\
P_{2}=-2 x_{3}, \\
P_{3}=-2 x_{2},
\end{array}\right\}
$$

then we have

$$
d_{H}\left(F_{i}\right)=P_{i} F_{i}, \quad i=1,2,3 .
$$

In order to prove the theorem, we show that (9) are all irreducible Darboux polynomials of the system.

For $f, g \in \mathbb{C}\left[\curvearrowleft_{\nVdash}, \ldots, \curvearrowleft_{\ltimes}\right]$ we write $f \propto g$ if there exists $a \in \mathbb{C}, a \neq 0$, such that $f=a g$. Note that every 'eigenvalue' $P$ for the Halphen system must be linear, i.e., it has the form $P=p_{1} x_{1}+p_{2} x_{2}+p_{3} x_{3}$.

Now we show the following

Lemma 2 If $F \in \mathbb{C}\left[\curvearrowleft_{\nVdash}, \curvearrowleft_{\not \prime}, \curvearrowleft_{\nVdash}\right] \backslash \mathbb{C}$ is a irreducible homogeneous polynomial of degree $m$ such that $d_{H}(F)=P F$, and $P=\alpha P_{i}$ for some $i \in\{1,2,3\}$, and $\alpha \in \mathbb{C}$ then $F \propto F_{i}$ and $\alpha=1$.

\section{Proof.}

First, assume that $i=1$ and introduce new variables

$$
z_{1}=x_{1}-x_{2}, \quad z_{2}=x_{2}-x_{3}, \quad z_{3}=x_{1}+x_{3} .
$$

In the new variables the Halphen system has the form

$$
\frac{d z_{1}}{d t}=z_{1} l_{1}, \quad \frac{d z_{2}}{d t}=-z_{2} l_{2}, \quad \frac{d z_{3}}{d t}=\frac{1}{2} l_{1} l_{2}
$$

where

$$
l_{1}=z_{1}+z_{2}-z_{3}, \quad l_{2}=z_{1}+z_{2}+z_{3} .
$$

Darboux polynomials and their corresponding 'eigenvalues' (9) in terms of the new variables have the form

$$
\left.\begin{array}{ll}
F_{1}=z_{1}, & F_{2}=z_{2}, \\
P_{1}=l_{1}, & F_{2}=-z_{1}-z_{2}, \\
P_{2}, & P_{3}=l_{3}=z_{1}-z_{2}-z_{3} .
\end{array}\right\}
$$


Let us assume that there exists an irreducible homogeneous Darboux polynomial $F$ of degree $m \geq 1$ with the 'eigenvalue' $P=p_{1} z_{1}+p_{2} z_{2}+p_{3} z_{3}=\alpha l_{1}$ for some $\alpha \in \mathbb{C}$, i.e.,

$$
z_{1} l_{1} \partial_{1} F-z_{2} l_{2} \partial_{2} F+\frac{1}{2} l_{1} l_{2} \partial_{3} F=P F ; \quad \partial_{i}=\frac{\partial}{\partial z_{i}}, \quad i=1,2,3 .
$$

Using the Euler identity for $F$, we can eliminate $\partial_{3} F$ from the above equation and, taking into account (10), we obtain

$$
z_{1} l_{1}^{2} \partial_{1} F+z_{2} l_{2}^{2} \partial_{2} F=\left(m l_{1} l_{2}-2 z_{3} P\right) F .
$$

We put $z_{1}=0$ in the above equation. The obtained equation has the form

$$
z_{2} \tilde{l}_{2}^{2} \partial_{2} \tilde{F}=\left(m \tilde{l}_{1} \tilde{l}_{2}-2 z_{3} \tilde{P}\right) \tilde{F}
$$

where we denote $\tilde{G}\left(z_{2}, z_{3}\right)=G\left(0, z_{2}, z_{3}\right)$ for an arbitrary polynomial $G$. Indeed, $\tilde{\partial_{2}} F=\partial_{2} \tilde{F}$. Now suppose that $F \not<z_{1}$. Under this assumption $\tilde{F} \neq 0$ because $F \neq 0$ is homogeneous and irreducible and thus $\operatorname{deg} \tilde{F}=\operatorname{deg} F=m$. From (13) we obtain

$$
\frac{\partial_{2} \tilde{F}}{\tilde{F}}=-\frac{m+2 p_{3}}{z_{2}}+\frac{2\left(m+p_{3}\right)}{z_{2}+z_{3}}-\frac{2\left(p_{2}-p_{3}\right) z_{3}}{\left(z_{2}+z_{3}\right)^{2}} .
$$

For fixed $z_{3} \neq 0$ it represents decomposition (7), and, as a consequence, we obtain that

$$
p_{2}-p_{3}=2 \alpha=0
$$

thus $P=0$. However, in such a case, from Lemma 1 we know that $F$ is constant. This contradicts the assumption that $\operatorname{deg} F \geq 1$. Consequently, $F \propto z_{1}$ and thus $m=1$. This ends the proof when $i=1$. Permutational invariance of the Halphen system (see (8)) implies that the same is true when $i=2$ or $i=3$.

Almost in the same way we prove the following

Lemma 3 If $F \in \mathbb{C}\left[\curvearrowleft_{\nVdash}, \curvearrowleft_{\not}, \curvearrowleft_{\nVdash}\right] \backslash \mathbb{C}$ is an irreducible homogeneous polynomial of degree $m$ such that $d_{H}(F)=P F$, and $F \not F_{1}, F \not F_{2}$ then $P=\alpha P_{3}, \alpha \in \mathbb{C}$.

Proof. Let us take $P=p_{1} z_{1}+p_{2} z_{2}+p_{3} z_{3}$ in equation (12). From the proof of the previous lemma we obtain that $p_{2}=p_{3}$ (see (14)). Next, put $z_{2}=0$ in equation (12). We obtain that

$$
z_{1} \hat{l}_{1}^{2} \partial_{1} \hat{F}=\left(m \hat{l}_{1} \hat{l}_{2}-2 z_{3} \hat{P}\right) \hat{F}
$$

where now for an arbitrary polynomial $G$ we denote $\hat{G}=G\left(z_{1}, 0, z_{3}\right)$. Under our assumption $\hat{F} \neq 0$ because $F \neq 0$ is homogeneous and irreducible and thus $\operatorname{deg} \hat{F}=\operatorname{deg} F=m$. From (15) we obtain

$$
\frac{\partial_{1} \hat{F}}{\hat{F}}=-\frac{m+2 p_{3}}{z_{1}}+\frac{2\left(m+p_{3}\right)}{z_{1}-z_{3}}-\frac{2\left(p_{1}+p_{3}\right) z_{3}}{\left(z_{1}-z_{3}\right)^{2}} .
$$


This implies that necessarily $p_{1}=-p_{3}$ and, consequently,

$$
P=-p_{3}\left(z_{1}-z_{2}-z_{3}\right)=-p_{3} l_{3}
$$

But $l_{3}$ is equal to $P_{3}$ expressed in variables $\left(z_{1}, z_{2}, z_{3}\right)$ (see ([1])).

Now we can proceed to the proof of our theorem stated in Introduction.

Proof. Let us assume that the Halphen system (1) possesses a rational first integral $F=A / B$, with relatively prime $A, B \in \mathbb{C}\left[\curvearrowleft_{\nVdash}, \curvearrowleft_{\sharp}, \curvearrowleft \nVdash\right]$. From point (1) in section 2 we know that polynomials $A$ and $B$ are Darboux polynomials corresponding to the same 'eigenvalue' $P \in \mathbb{C}\left[\curvearrowleft_{\nVdash}, \curvearrowleft_{\sharp}, \curvearrowleft_{\nVdash}\right]$. From Lemma 1 we deduce that $P \not \equiv 0$. From Lemmas 2 and 3 we deduce that the only irreducible homogeneous Darboux polynomials of the Halphen system are those given by (9). Let us observe that the linear forms $P_{i}, 1 \leq i \leq 3$ are linearly independent, and thus, from the point (6) of the previous section, we deduce that all the Darboux polynomial of the Halphen system are monoms $\alpha F_{1}^{i_{1}} F_{2}^{i_{2}} F_{3}^{i_{3}}$ for some non-negative integers $i_{1}, i_{2}, i_{3}$ such that $i_{1}+i_{2}+i_{3}>0$, and $\alpha \in \mathbb{C}$. It follows that both $A$ and $B$ are of this form. As they corresponds to the same 'eigenvalue' $P$, by Lemmas 2 and 3 they proportional and thus they are not relatively prime as was assumed. Contradiction shows that our theorem is true.

\section{Acknowledgments}

We sincerely thank A. Nowicki from Department of Mathematics of N. Copernicus University for many inspiring discussions. For the first author this work was supported also by grant KBN 22149203.

The second author acknowledges the Institutes of Astronomy and of Mathematics of Torun University for their hospitality and excellent working conditions during August 1994, when this work was done.

\section{References}

[1] S. Chakravarty. A class of integrable conformally self-dual metrics. Classical Quantum Gravity, 11:L1-L6, 1994.

[2] S. Chakravarty, M. J. Ablowitz, and P. A. Clarkson. Reduction of self-dual Yang-Mills fields and classical systems. Phys. Rev. Lett., 65(9):1085-1087, 1993.

[3] G. Darboux. Mémoire sur les équations différentielles algèbriques du premier ordre et du premier degré. Bull. Sci. Math. 2ème série, 2:60-96, 123-144, 151-200, 1878. 
[4] V. A. Dobrovol'skii, N. V. Lokot', and J.-M. Strelcyn. Mikhail Nikolaevich Lagutinskii (1871-1915), un mathématicien méconnu. Preprint, pages 1-35, 1993.

[5] V. A. Dobrovol'skii, A. J. Maciejewski, and J.-M. Strelcyn. The mathematical works of Mikhail Nikolaevich Lagutinskii (1871-1915). in preparation.

[6] A. R. Forsyth. The Theory of Differential Equations, volume I-VI. Cambridge University Press, Cambridge 1890-1902 reprinted by Dover Publications, New York, 1959.

[7] H. Grümal and Y. Nutku. Poisson structure of dynamical systems with three degrees of freedom. J. Math. Phys., 34(12):5691-5723, 1993.

[8] G.-H. Halphen. Sur un système d'equations différentielles. C. R. Acad. Sci., 92:1101-1103, 1881.

[9] G.-H. Halphen. Sur certains système d'equations différentielles. C. R. Acad. Sci., 92:1404-1407, 1881.

[10] J.-P. Jouanolou. Équations de Pfaff algébriques, volume 708 of Lectures Notes in Mathematics. Springer-Verlag, Berlin, 1979.

[11] M. Kummer, R. C. Churchill, and D. L. Rod. On a result of Bruns. Canad. Math. Bull., 33(2):175-180, 1990.

[12] M. N. Lagutinskii. Partial Algebraic Integrals. Kharkov, 1908. in Russian.

[13] M. N. Lagutinskii. Application of the polar operations to integration of ordinary differential equations in the finite form. Soobshch. Kharkov. Mat. Obshch., ser. 2, XII:111-243, 1911. in Russian.

[14] J. Moulin Ollagnier, A. Nowicki, and J.-M. Strelcyn. On the non-existence of constants of derivations: the proof of a theorem of Jouanolou and its development. Bull. Sci. Math. (2), 1995. to appear.

[15] A. Nowicki. On the non-existence of rational first integrals for system of linear differential equations. J. Pure Appl. Algebra, 1994. to appear.

[16] A. Nowicki. Polynomial derivations and their rings of constants. Nicolaus Copernicus University Press, Toruń, 1994.

[17] I. A. B. Strachan. Integrable and near-integrable dynamical systems associated with Bianchi IX matrics. Teoret. Mat. Fiz., 99(3):545-551, 1994.

[18] L. A. Takhtajan. A simple example of modular forms as tau-functions for integrable equations. Teoret. Mat. Fiz., 93(2):330-341, 1992. 
[19] L. A. Takhtajan. Modular forms as $\tau$-functions for certain integrable reduction of the Yang-Mills equations. In O. Babelon, P. Cartier, and Y. Kosmann-Schwarzbach, editors, Integrable Systems. The Verdier Memorial Conference. Actes du Colloque Intenational de Luminy, pages 115-129, Berlin, 1993. Birkhäuser.

[20] L. A. Takhtajan. On foundation of the generalized Nambu mechanics. Comm. Math. Phys., 160:295-315, 1994.

[21] S. L. Ziglin. Branching of solutions and non-existence of first integrals in Hamiltonian mechanics. I. Functional Anal. Appl., 16:181-189, 1982. 